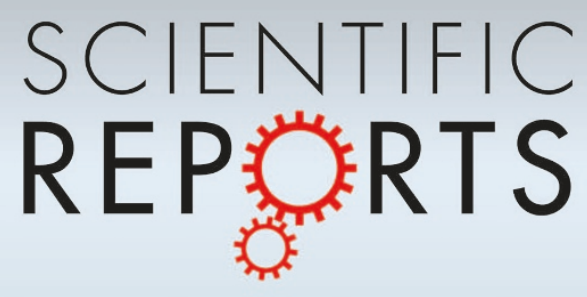

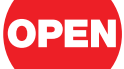

SUBJECT AREAS:

MATERIALS SCIENCE

PHYSICS

MAGNETIC MATERIALS AND

DEVICES

APPLIED PHYSICS

Received

29 February 2012

Accepted

7 June 2012

Published

28 June 2012

Correspondence and requests for materials should be addressed to A.S. (slavin@oakland. edu)

\section{Nano-structured magnetic metamaterial with enhanced nonlinear properties}

\author{
Yuri Kobljanskyi' , Gennady Melkov' ${ }^{1}$ Konstantin Guslienko ${ }^{2,3}$, Valentyn Novosad ${ }^{4}$, Samuel D. Bader ${ }^{4}$, \\ Michael Kostylev ${ }^{5} \&$ Andrei Slavin ${ }^{6}$
}

\begin{abstract}
${ }^{1}$ Faculty of Radiophysics, Taras Shevchenko National University of Kyiv, Kyiv 01601, Ukraine, ${ }^{2}$ Departamento de Física de Materiales, Universidad del País Vasco, UPV/EHU, 20018 San Sebastian, Spain, ${ }^{3}$ IKERBASQUE, The Basque Foundation for Science, 48011 Bilbao, Spain, ${ }^{4}$ Materials Science Division, Argonne National Laboratory, Argonne, Illinois 60439, USA, ${ }^{5}$ School of Physics, M013, University of Western Australia, Crawley, WA 6009, Australia, ${ }^{6}$ Department of Physics, Oakland University, Rochester, MI 48309, USA.
\end{abstract}

Nano-structuring can significantly modify the properties of materials. We demonstrate that size-dependent modification of the spin-wave spectra in magnetic nano-particles can affect not only linear, but also nonlinear magnetic response. The discretization of the spectrum removes the frequency degeneracy between the main excitation mode of a nano-particle and the higher spin-wave modes, having the lowest magnetic damping, and reduces the strength of multi-magnon relaxation processes. This reduction of magnon-magnon relaxation for the main excitation mode leads to a dramatic increase of its lifetime and amplitude, resulting in the intensification of all the nonlinear processes involving this mode. We demonstrate this experimentally on a two-dimensional array of permalloy nano-dots for the example of parametric generation of a sub-harmonic of an external microwave signal. The characteristic lifetime of this sub-harmonic is increased by two orders of magnitude compared to the case of a continuous magnetic film, where magnon-magnon relaxation limits the lifetime.

W hen the lateral sizes of a magnetic particle decrease below a micron, its properties are modified due to geometrical confinement and size effects ${ }^{1,2}$. In particular, the ground-state magnetization distribution can be either spatially uniform or vortex-like, depending on the particle aspect ratio ${ }^{3}$. The frequency spectrum of spin-wave excitations in a magnetic particle can also be drastically changed. The modification of the spin wave excitation spectra due to the boundary conditions imposed by the edges of magnetic nano-particles leads to a spectral quantization and elimination of excitations that have half-wavelengths larger than the particle size. The quantization of the excitation spectrum of small magnetic particles was observed experimentally using different techniques ${ }^{4}$. The discrete values of the spin wave eigenfrequencies are mainly determined by the magnetostatic interaction and depend on the particle lateral sizes and magnetization static configuration (the ground state $)^{5,6}$. The discretization of the spin-wave spectrum related to the reduction of the particle sizes can reduce and even remove the frequency degeneracy between the main excitation mode (spatially quasi-uniform ferromagnetic resonance (FMR) mode) and the spin-wave modes with higher values of the in-plane wave vector ${ }^{7}$ and, therefore, can substantially reduce the strength of various multi-magnon relaxation processes related to this degeneracy. The critical issue is the removal of degeneracy of the FMR mode with spin wave modes having the wavenumber of the order of $10^{4} \mathrm{~cm}^{-1}$ and the lowest magnetic damping (lower than the damping of the FMR mode $^{6}$ ). The suppression of the magnon-magnon relaxation for the pumped ferromagnetic resonance mode leads to a dramatic increase of its life-time, amplitude, and, consequently, to an increase of the intensity of all the nonlinear processes involving this mode $e^{6-8}$.

In this work we demonstrate that nano-structuring of a magnetic material leads to a drastic increase of a lifetime of the main ferromagnetic resonance mode parametrically excited by an external microwave pumping signal. The effect is demonstrated experimentally in a two-dimensional array of permalloy nano-dots subjected to the action of a spatially uniform microwave pumping field having the frequency that is twice larger than the frequency of the ferromagnetic resonance mode in an individual magnetic nano-dot.

Figure $1(a, b, c)$ demonstrates the qualitative modification of the spin wave spectrum of a finite-size magnetic element (in particular, of a cylindrical magnetic dot of the thickness $L$ and radius $R^{5,9}$ ) when the element size is reduced. Figure 1 (d) provides an example of the numerical calculation of the spin wave eigenfrequencies for a Permalloy dot or the $L=10 \mathrm{~nm}$ and $R=100 \mathrm{~nm}$ performed $\mathrm{in}^{5}$. The spectral modifications similar to the ones illustrated in Fig. 1 (a,b,c) will take place for magnetic elements of any shape made from both ferromagnetic 
a Film

b Dot $\mathrm{R}=1000 \mathrm{~nm}$
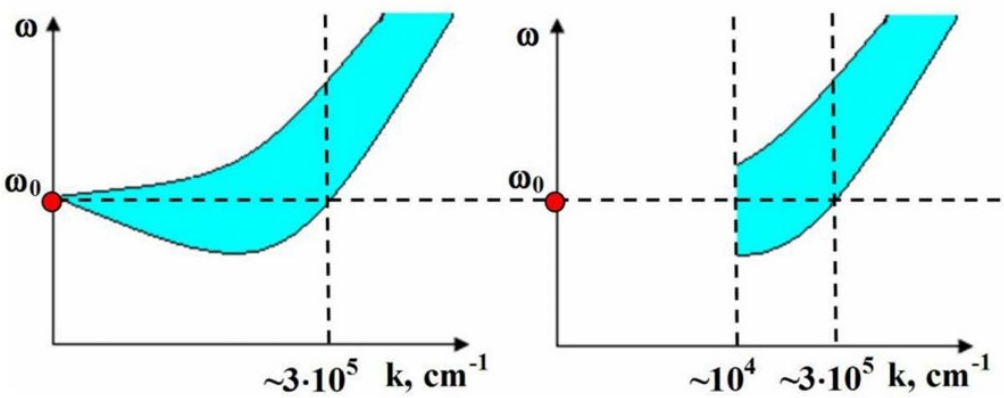

c Dot $\mathrm{R}<100 \mathrm{~nm}$

d Dot $\mathrm{R}=100 \mathrm{~nm}$

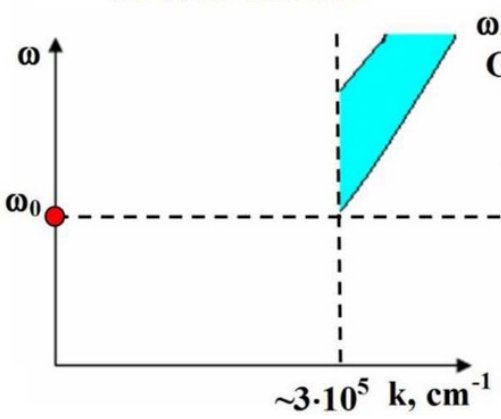

$\omega / 2 \pi$,

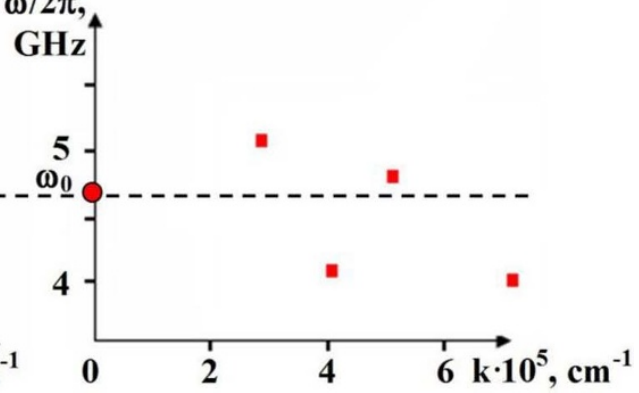

Figure 1 Spin-wave spectrum of a finite-size magnetic nano-element. The qualitative picture of the spin-wave spectrum: (a) of a continuous magnetic film, (b) of a magnetic dot having the radius $R=1000 \mathrm{~nm}$, and thickness $L \ll R$, (c) of a dot having $R<100 \mathrm{~nm}(L \ll R)$. The angular frequency of the quasi-uniform FMR mode is denoted as $\omega_{0}$. Panel $(d)$ shows the results $(\boldsymbol{\square})$ of a quantitative calculation (presented in Ref. ${ }^{5}$ ) of the frequencies of all spinwave modes in an in-plane magnetized (bias magnetic field $H_{0}=300 \mathrm{Oe}$ ) cylindrical Py dot of the radius $R=100 \mathrm{~nm}$ and thickness $L=10 \mathrm{~nm}$ that differ from the frequency $\omega_{0}$ of the FMR mode by less than $500 \mathrm{MHz}$.

metals and dielectrics. The frequency of the ferromagnetic resonance (FMR) $\omega_{0}$, corresponding to the spatially uniform precession of magnetization with wave number $k=0$ for most magnetic elements can be approximately evaluated using the model of an equivalent ellipsoid, for which the effective demagnetization factors $N_{x}, N_{y}, N_{z}$ are determined by the aspect ratio of the particle $(R / L$ in the case of a cylindrical dot $)^{10}$.

In the following we will consider a thin cylindrical magnetic dot of radius $R$ and thickness $L \ll R$. For a thin $\operatorname{dot}(L \ll R)$ in the $x z$ plane, magnetized to saturation along the $z$ axis by the bias magnetic field $H_{0}$, the FMR frequency is ${ }^{6}$ :

$$
\omega_{0}=\gamma \sqrt{H_{0}\left[H_{0}+\left(N_{y}-N_{z}\right) M_{0}\right]}
$$

where $\gamma$ is the gyromagnetic ratio, and $M_{0}$ is the saturation magnetization. The approximation of a disk-shaped particle by an ellipsoid is quantitatively correct only in the limit $L \ll R$. In a real situation the internal, static magnetic field in the disk becomes non-uniform and the spatial distribution of the FMR mode becomes quasi-uniform, which leads to a slight increase of its frequency $\omega_{0}$ with decreasing $R^{11,12}$.

A decreasing dot radius has a strong effect on the spatially nonuniform, higher spin-wave modes having wave numbers $k>0$. Due to the influence of the boundary conditions at the dot lateral edges the long-wavelength part of the spin wave spectrum is depleted, since the dot can only support modes having half-wavelengths $\lambda / 2 \leq R$. Thus, the spin waves with wave number $k=2 \pi / \lambda \leq \pi / R$ will be eliminated from the dot spectrum (see Fig. $1 b, c$ ). Also, due to the confinement of the dot size along the three Cartesian coordinates, the spectrum becomes discrete. The exact calculation of discrete spin wave eigenfrequencies of a magnetic dot has been performed ${ }^{5,9}$. An example of such a discrete spectrum for an in-plane magnetized $\left(H_{0}\right.$ $=300 \mathrm{Oe}$ ) permalloy (Py) dot calculated in ${ }^{5}$ is presented in Fig. $1 \mathrm{~d}$ for
$R=100 \mathrm{~nm}$ and $L=10 \mathrm{~nm}$ (denoted $100 \times 10)$, showing the positions of all the eigen-modes within $\pm 500 \mathrm{MHz}$ of $\omega_{0}$. It can be seen in Fig. $1 d$ that the frequency degeneracy between the FMR mode and higher- $k$ spin wave modes can be eliminated, and that there are only five modes present.

Each of these features (the depletion of the long-wave part of the spectrum, the spectrum discretization, and the lifting of the frequency degeneracy) in the dots can modify the nonlinear dynamic response on a nano-structured magnetic material compared to that of a bulk material. In natural bulk magnetic materials the amplitude of the FMR mode is usually limited by four-wave ( $2^{\text {nd }}$ order) parametric nonlinear processes involving the higher spin-wave modes degenerate in frequency with the FMR mode ${ }^{6,8}$. Nano-structuring of the magnetic material and the related modification of the spin wave spectrum can remove the frequency degeneracy between the FMR mode and the higher spin wave modes, and can lead to a substantial increase in the lifetime and amplitude of the main FMR mode.

To prove this experimentally, we studied the process of parametric generation of a sub-harmonic of an external microwave signal with the frequency $\omega_{p}$ (such that $\left.\omega_{0}=\omega_{p} / 2\right)$ in an artificial magnetic meta-material formed by a planar array of non-interacting (interdot distance $d \gg L$, patterned area $S \sim 1 \mathrm{~mm}^{2}$ ) cylindrical Py nano-dots (see Fig. 2). The parametric excitation of spin waves and oscillations in the array occurs via the method of parallel field pumping ${ }^{6,8}$, where the magnetic field $h_{p}$ of the external microwave pumping signal $h_{p}=h \cos \left(\omega_{p} t\right)$ is applied parallel to the direction of the bias magnetic field $\mathrm{H}_{0}$. When the value of the pumping field amplitude $h$ exceeds a mode-dependent threshold value $h_{t h}^{(k)}{ }^{6}$, the amplitude of the spin wave mode, having the sub-harmonic frequency $\omega_{k}=\omega_{p} / 2$, starts to increase exponentially. The rate of this exponential growth is proportional to the super-criticality $\zeta=h / h_{t h}^{(k)}{ }_{8,13}$. Thus, the mode 


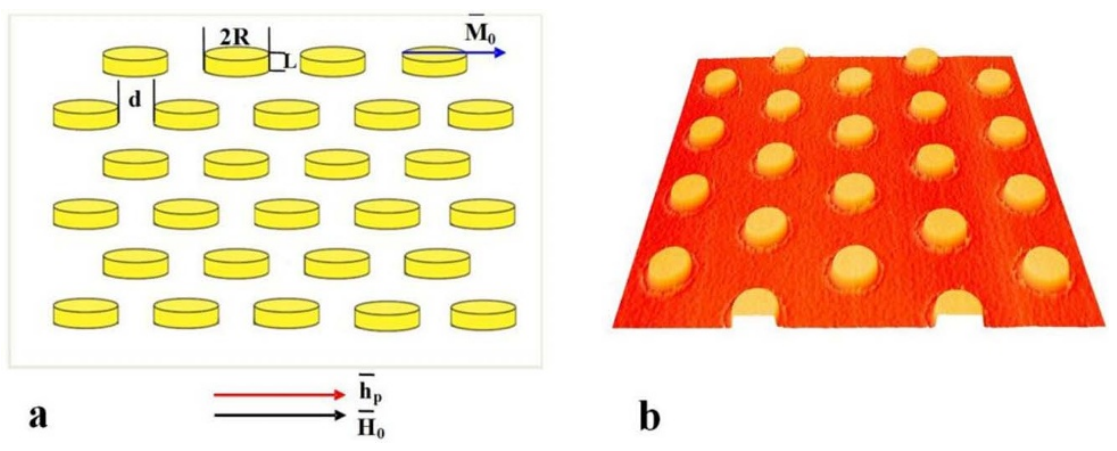

Figure 2 Two-dimensional array of cylindrical magnetic dots as a novel magnetic metamaterial. (a) Geometric and magnetic parameters of the array: $R$ and $L$ are the dot radius and thickness, respectively, $d$ is the distance between the dot edges, $\mathbf{M}_{0}$ is the saturation magnetization, $\mathbf{H}_{0}$ is the in-plane bias magnetic field, and $\mathbf{h}_{\mathrm{p}}$ is the magnetic field of microwave parallel pumping, $(b)$ Experimental picture of one of the studied arrays of Py dots $(R=1000 \mathrm{~nm}$, $L=12 \mathrm{~nm}$ ) obtained utilizing atomic force microscopy.

having the lowest excitation threshold $h_{t h}^{(k)}=h_{t h}^{\min }$ has the largest growth rate and, therefore, the largest amplitude. This dominant spin wave mode, through four-wave (2nd order) nonlinear interaction processes, starts to suppress all the other pumped modes and ultimately is the only one to survive ${ }^{8}$. All the other modes for which $h_{t h}^{(k)}>h_{t h}^{\min }$ decay exponentially until they are completely suppressed $^{14}$. In bulk magnets and continuous films ${ }^{6,15}$ the minimum parametric threshold corresponds to spin waves having $k \sim 10^{4}$ $\mathrm{cm}^{-1}$. It is these waves that suppress all the other spin waves and oscillations, including the quasi-uniform FMR mode that has $h_{t h}^{(0)}>h_{t h}^{\min }$.

It is clear from Fig. $1 \mathrm{~b}$ that when the radius of a dot is reduced to $R \sim 1 \mu \mathrm{m}$, the waves with $k \sim 10^{4} \mathrm{~cm}^{-1}$ are eliminated from the spectrum and the FMR mode becomes the dominant mode having the lowest threshold of parametric excitation. This statement is supported by the results of the recent experiment performed by means of the Brillouin light scattering spectroscopy ${ }^{11}$ : the quasi-uniform FMR mode, indeed, has the threshold of parametric excitation that is 3-10 dB lower than the threshold of excitation of higher- $k$ spin-wave modes (see Fig. 2 in Ref. ${ }^{11}$ ). In such a case only the dominant FMR mode will survive in the parametric excitation process, and this mode will suppress all other modes via the four-wave processes of nonlinear spin-wave interaction.

Previous experiments of parametric sub-harmonic generation in magnetic films ${ }^{15}$ have shown that the amplitude of the FMR mode of frequency $\omega_{0}=\omega_{p} / 2$, that initially grew under the action of parametric pumping, decayed exponentially as soon as the amplitudes of low-threshold spin waves with $k \sim 10^{4} \mathrm{~cm}^{-1}$ growing from the thermal level became sufficiently large ${ }^{15}$. As a result of this nonlinear suppression of the FMR mode, the electromagnetic radiation at the sub-harmonic frequency caused by the FMR is observed only during a short time interval ( $\sim 200 \mathrm{~ns})$ after the pumping is switched on. The dominant spin waves with $k \sim 10^{4} \mathrm{~cm}^{-1}$ excited by parametric pumping at the same frequency $\omega_{k}=\omega_{p} / 2$ do not contribute to the sub-harmonic electromagnetic radiation due to the large wave number mismatch between these waves and the electromagnetic waves having the same sub-harmonic frequency. Thus, in a nanostructured magnetic material (e.g. in an array of magnetic dots having $R \leq 1 \mu \mathrm{m}$ ), where the low-threshold spin waves with $k \sim 10^{4} \mathrm{~cm}^{-1}$ are eliminated from the spectrum, one can expect a significant increase of the time interval in which the FMR mode creates electromagnetic radiation at the sub-harmonic frequency.

\section{Results}

To prove these ideas experimentally we developed the set-up shown in Fig. 3 for the investigation of parametrically induced subharmonic generation in both continuous and patterned films. The sample (1) (either a 2D array of nano-dots or a continuous film) on the dielectric substrate (4) is placed inside an open dielectric resonator (2) made of ceramic with dielectric constant $\varepsilon \cong 80$. The external microwave pumping field has the frequency $\omega_{p} / 2 \pi=9.4 \mathrm{GHz}$. The microwave magnetic field $\mathbf{h}_{\mathrm{p}}$ created in the dielectric resonator was oriented along the plane of the sample and was parallel to the in-plane bias magnetic field $\mathrm{H}_{0}$ (the geometry of "parallel" parametric pumping ${ }^{6,8}$ ). The short-circuited antenna (3) made of $50-\mu \mathrm{m}$ diameter $\mathrm{Cu}$ wire was used to supply to the experimental sample (1) a short, synchronizing external signal of the power $P_{\text {in }}$ and to receive an output signal $P_{\text {out }}$ of electromagnetic radiation from the sample. Both the signals are at $4.7 \mathrm{GHz}$ (half of the pumping frequency) and were separated using a Y-circulator (see Fig. 3). The external synchronizing signal $P_{\text {in }}$ guaranteed the same initial phase for the FMR sub-harmonic oscillations parametrically excited by pumping in all the magnetic dots, thus creating the constructive interference of all oscillations, resulting in a coherent macroscopic output electromagnetic signal of the power $P_{\text {out }}$.

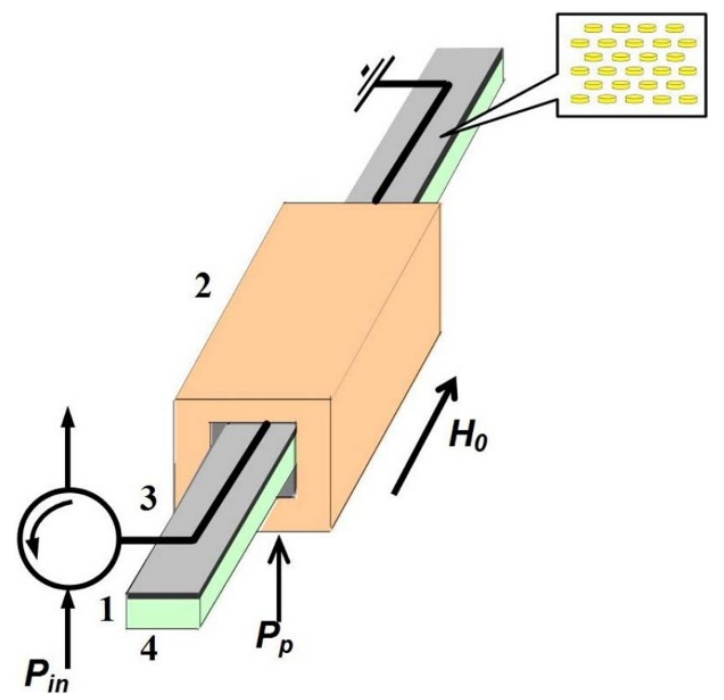

Figure 3 Experimental setup. $1-2 \mathrm{D}$ array of Py dots (1.6 $\mathrm{mm}$ by $3.0 \mathrm{~mm}), 2$ - open dielectric resonator for the supply of microwave pumping, 3- wire microwave antenna (diameter $50 \mu \mathrm{m}$ ), 4- nonconductive GaAs substrate, $P_{p}$ is the pumping power, $P_{i n}$ is the power of the input synchronizing signal, $P_{\text {out }}$ is the power of the output signal created by the sub-harmonic radiation from the dot array, and $\mathbf{H}_{0}$ is the in-plane bias magnetic field. 
The samples were 2D arrays of cylindrical non-interacting Py dots (see Fig. 2) having the same radius $R=1000 \mathrm{~nm}$, the same distance $d=1000 \mathrm{~nm}$ between the dot lateral edges, and two different thicknesses: $L_{1}=100 \mathrm{~nm}$ (dot array \#1) and $L_{2}=12 \mathrm{~nm}$ (dot array \#2) formed on a non-conductive GaAs substrate of thickness $0.5 \mathrm{~mm}$ (see Methods). As a control we used an unpatterned, continuous Py film of the thickness $100 \mathrm{~nm}$ on the same GaAs substrate.

The samples were subjected at $t=0$ to the simultaneous action of a long $\left(\tau_{p}=9 \mu \mathrm{s}\right)$ and powerful (the power $P_{p} \sim 1-100 \mathrm{~W}$ ) pulse of microwave parallel pumping field, and a short ( $\left.\tau_{\text {in }}=30 \mathrm{~ns}\right)$ and relatively weak $\left(P_{\text {in }} \sim 10 \mu \mathrm{W}\right)$ pulse of synchronizing microwave signal (see details in Methods). The $P_{\text {out }}$ signal was received by the antenna (3). As expected, the output signal at the antenna (3) appeared only when the pumping power exceeded the threshold $P_{p}^{\text {th }}$ of parametric excitation of the FMR mode, which in both the dot arrays was around $P_{p}^{\text {th }} \cong 20 \mathrm{~W}$. The output power $P_{\text {out }}$ increased with increase of the pumping power from the threshold value to the maximum available value of $P_{p}=100 \mathrm{~W}$. The maximum value of $P_{\text {out }}$ was obtained by tuning the bias magnetic field $H_{0}$ to achieve the resonance condition of the FMR mode $\omega_{0}$ with the pumping sub-harmonic $\omega_{p} / 2$ : $\omega_{0}=\omega_{p} / 2$.

The experimentally measured time dependences of the power of microwave radiation $P_{\text {out }}(t)$ with the sub-harmonic frequency $\omega_{0}=\omega_{p} / 2$ for all three samples are presented in Fig. 4 . It is seen from Fig. 4, that when the parametric pumping field is switched on at $t=0$, the output power $P_{\text {out }}$, proportional to the intensity of the radiated sub-harmonic microwave signal, starts to increase exponentially. The theory of parametric excitation ${ }^{8,13}$ predicts an exponential increase of the output power with time increasing described by the expression $P_{\text {out }} \sim \exp \left\{2 \Gamma_{0}\left[\sqrt{P_{p} / P_{p}^{\text {th }}}-1\right] t\right\}\left(\Gamma_{0}\right.$ is the relaxation frequency of the FMR mode) until this power reaches a maximum level determined by the four-magnon phase mechanism of power limitation ${ }^{8}$. In the experiment (Fig. 4) this level is reached after $t=200-300 \mathrm{~ns}$. Also, we see that the output power in a continuous film grows faster than in both the dot arrays investigated. This is due to the higher threshold of parametric excitation of the spin waves in the dot arrays caused by the increase of the relaxation frequency in the dot arrays due to patterning. The temporal evolution of the output power in a continuous film and arrays of nano-dots is very

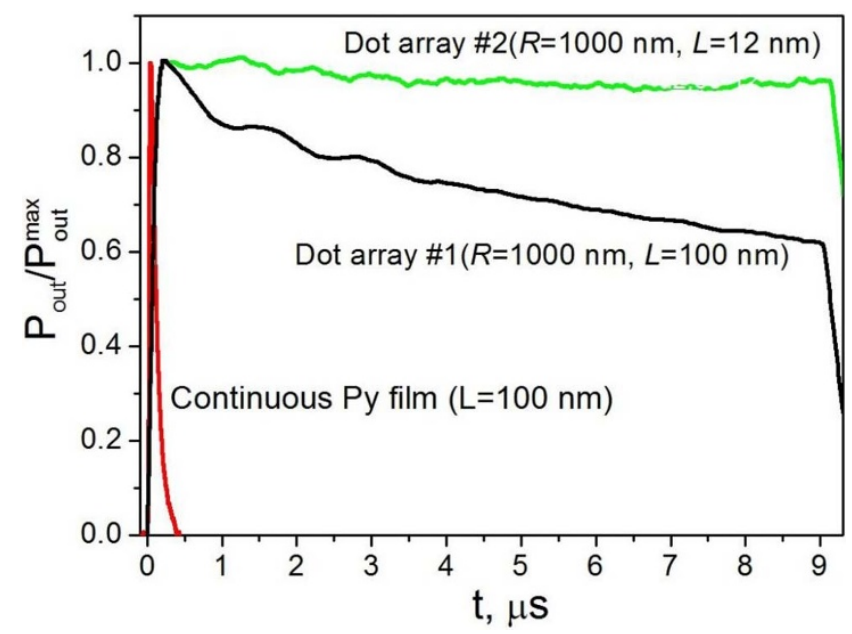

Figure 4 | Experimental dependence of the normalized output power $P_{\text {out }} / P_{\text {out }}^{\max }$ on the time of action of the microwave pumping. black line continuous Py film of the thickness $L=100 \mathrm{~nm}, H_{0}=260$ Oe, red line dot array $\# 1(R=1000 \mathrm{~nm}, L=100 \mathrm{~nm}), H_{0}=300$ Oe, green line - dot array $\# 2(R=1000 \mathrm{~nm}, L=12 \mathrm{~nm}), H_{0}=300$ Oe. Parameters of the pumping and signal pulses: $P_{p}=100 \mathrm{~W}, \tau_{p}=9 \mu \mathrm{s}, \omega_{p} / 2 \pi=9.4 \mathrm{GHz}$; $P_{\text {in }}=10 \mu \mathrm{W}, \tau_{\text {in }}=30 \mathrm{~ns}, \omega_{\text {in }} / 2 \pi=4.7 \mathrm{GHz}$. different. As explained above, in a continuous film, the influence of the low-threshold spin waves with $k \sim 10^{4} \mathrm{~cm}^{-1}$ leads to the rapid exponential decrease the output power $P_{\text {out }}$ after the time interval $\tau \approx 200 \mathrm{~ns}$.

In contrast, in the case of $2 \mathrm{D}$ arrays of magnetic dots of the thickness $L=100 \mathrm{~nm}$ the decrease of the output power with time is approximately two orders of magnitude slower than in the case of a continuous magnetic film (see Fig. 4). Also from Fig. 4, we see that with decrease of the dot thickness to $L=12 \mathrm{~nm}$, the decrease of the output power with time gets even slower. These are the main experimental results obtained in our paper. Similar results - a slow decrease of the power with time - were obtained on the dot arrays with the sizes $R \times L$ of $900 \times 50,1000 \times 40$, and $1000 \times 12$ (all the sizes are in $\mathrm{nm}$ ).

We attribute the dramatic difference in the temporal evolution of the parametrically excited, sub-harmonic radiation in the patterned dot arrays $v s$. continuous films to the elimination from the dot spectrum of spin-waves degenerate in frequency with the FMR mode and having $k \leq 10^{4} \mathrm{~cm}^{-1}$. The threshold of parametric excitation of the quasi-uniform FMR mode then becomes lower than that of the other modes. In such a case the theory of parametric excitation ${ }^{8}$ predicts that in the stationary regime the amplitude of the parametrically excited FMR mode, and, therefore, the output power $P_{\text {out }}$, should remain constant during the action of the pumping pulse.

In the experiment shown in Fig. 4 we see that for the dot array $1000 \times 12$ (sample \#2) the output power is, indeed, nearly constant in the whole temporal interval of the microwave pumping action, while in the array $1000 \times 100$ (sample \#1) the output power slowly decreases with time increasing, characterized by a time constant of the order of $\tau \sim 10 \mu \mathrm{s}$, which is $2-3$ orders of magnitude longer than typical times of the magnon-magnon relaxation ${ }^{6,16}$. We believe that the slow decrease of the power $P_{\text {out }}$ results from the heating of the dots caused by the absorption of pumped microwave power. In such a case, the temperature change would be proportional to the dot thickness, and the corresponding time constant would be of the order of $\tau \sim 10 \mu \mathrm{s}^{17-19}$ (see Methods), in agreement with the experimental data shown in Fig. 4.

\section{Discussion}

In this article we have studied the influence of magnetic particle sizes on its non-linear dynamic properties. In bulk samples and continuous magnetic films the amplitude of the FMR mode is limited at a rather low level by the four-wave magnon-magnon interaction processes involving spin wave modes having large magnitudes of the wave number and frequencies that are close to the frequency of the FMR mode. We demonstrated above that in sufficiently small magnetic particles it should be possible to completely eliminate the frequency degeneracy of the FMR mode with short-wave spin wave modes and, therefore, to substantially increase the possible amplitude and life-time of the FMR mode excited in a magnetic particle. As a result, in a small magnetic particle the efficiency of all the nonlinear processes, such as the frequency multiplication, rectification, parametric amplification or/and generation, parametric wave front reversal, and Brillouin inelastic light scattering should be substantially increased.

The above results allow us to conclude, that for both $2 \mathrm{D}$ arrays of magnetic nano-dots studied in our experiments the nano-structuring of the magnetic material lead to the exclusion of the majority of fourwave processes of magnon-magnon relaxation limiting the amplitude of the quasi-uniform FMR mode in a continuous magnetic film and bulk magnetic samples. This exclusion resulted in a substantial enhancement of the nonlinear properties of the magnetic dot array at the frequency of the main FMR mode. In particular, this nanostructuring resulted in the drastic increase of the characteristic time of parametrically induced subharmonic radiation from the array by two orders of magnitude in comparison with the case of a continuous magnetic film of a similar thickness. The slow decrease of the 
radiated subharmonic power observed in Fig. 4 is caused by the microwave heating of magnetic dots and can be substantially reduced by reducing the dot thickness (compare curves for the dot arrays of the thickness $L=100 \mathrm{~nm}$ and $L=12 \mathrm{~nm}$ shown in Fig. 4).

In summary, we have proven experimentally that nanostructuring of a magnetic material can substantially enhance the nonlinear dynamic properties of the material. Thus, using the nanostructuring, it is possible to develop novel artificial metamaterial with nonlinear microwave properties that are superior to that of magnetic films and traditional bulk magnetic materials. These novel patterned meta-materials can be useful for applications in reciprocal (filters, oscillators), non-reciprocal (isolators, circulators) and nonlinear (detectors, frequency multipliers) microwave signal processing devices operating at high levels of microwave power.

\section{Methods}

Microfabrication. Permalloy $\left(\mathrm{Fe}_{20} \mathrm{Ni}_{80}\right)$ disks were defined on an undoped $<100>$ GaAs wafer by mean of photolithography and electron-beam evaporation techniques. The process starts with spin coating of positive tone $\$ 1813$ photoresist (Shipley Co) at $3,000 \mathrm{rpm}$ for $60 \mathrm{sec}$; followed by soft baking on a hot plate at $115^{\circ} \mathrm{C}$ for $90 \mathrm{sec}$. After exposure to 365-nm light, the sample was developed using a 1:5 mix of Microposit 351 (Microresist Technology $\mathrm{GmbH}$ ) and de-ionized water. Then, the electron beam evaporation of Py was performed at room temperature at a base pressure of $1 \times 10^{-8}$ Torr, with a deposition rate of $0.2 \mathrm{~A} / \mathrm{sec}$. The Py layer was topped with $2 \mathrm{~nm}$ of Ti in situ to prevent oxidation of the samples. Finally, an ultrasound-assisted lift-off in acetone completes the process.

Measurements. The dielectric resonator together with the experimental sample (2D array of Py dots or a continuous Py film) was placed in the maximum of the magnetic field $h_{p}$ of microwave pumping $\left(\mathbf{h}_{\mathfrak{p}} \| \mathbf{H}_{0}\right)$ inside a hollow metallic waveguide (wavelength $\lambda=3 \mathrm{~cm}$ ) carrying the $H_{10}$ mode (see Figs. 2,3). The plane of the sample was parallel to the wider wall of the waveguide. The impedance matching between the waveguide and the dielectric resonator, and the fine-tuning of the resonator frequency, was done by means of a piston short-circuiting the waveguide. The resonator was tuned to the frequency $\omega_{p}$ of the microwave pumping by minimizing the reflection from the resonator. The bias magnetic field $H_{0}$ was chosen to make the FMR frequency in the sample equal to $\omega_{p} / 2$. The input synchronizing signal of power $P_{i n}$, duration $30 \mathrm{~ns}$, and carrier frequency $\omega_{p} / 2$, was supplied to the wire antenna (see " 3 " in Fig. 3) through the circulator and a coaxial cable. This signal was only weakly absorbed by the experimental sample (absorption $\sim 1 \%$ ) and was nearly totally reflected, forming an image of the input synchronizing pulse on the oscilloscope. Simultaneous with the input signal, the microwave pumping pulse of the carrier frequency $\omega_{p}$ and duration $\tau_{p}=9 \mu$ s was supplied to the resonator. When the pumping power $P_{p}$ was lower than the threshold $P_{t h}$ of parametric generation of a pumping sub-harmonic, the only signal on the oscilloscope was the input synchronizing pulse. When the pumping power exceeded the threshold $P_{p}>P_{t h}$, an additional delayed signal, caused by the parametric radiation of the pumping subharmonic $\omega_{p} / 2$ from the sample, appeared on the oscilloscope. In the following experiments this additional signal was obtained by subtraction of the input synchronizing pulse from the total output signal.

Microwave absorption and heat exchange in Py dot formed on a non-conductive substrate. In the case of a thin cylindrical magnetic dot (with radius $R$ that is substantially larger than the thickness $L, L<<R$ ) formed on a solid, non-conductive substrate, the equation describing the dot heating due to the absorption of the external microwave pumping field can be derived from the first law of thermodynamics (or conservation of energy) which for the constant volume $V$ of the dot can be written as the equation of a heat balance:

$$
\Delta U=\Delta Q_{+}-\Delta Q_{-},
$$

where the change of the internal energy $\Delta U=c V \Delta T$ of the dot is equal to the difference between the amount of heat $\Delta Q_{+}$absorbed from the microwave pumping and the amount of heat $\Delta Q_{-}$radiated into the substrate, $c$ is the volume heat capacity of the dot material, and $\Delta T$ is the change in the dot temperature.

Taking a time derivative of the equation of the heat balance (1), the following equation describes the temporal evolution of the dot temperature:

$$
c V \frac{\partial \Delta T}{\partial t}=P_{a b s}-P_{\text {rad }} .
$$

Here the power $P_{a b s}$ absorbedby the dot is determined by the equation:

$$
P_{a b s}=\frac{\partial Q_{+}}{\partial t}=\frac{1}{2} \chi^{\prime \prime} \omega_{p} h_{p}^{2} V,
$$

where $\chi^{\prime \prime}$ is the imaginary part of the dimensionless dot magnetic susceptibility, $\omega_{p}$ is the angular frequency of microwave pumping, and $h_{p}$ is the amplitude of the pumping microwave magnetic field that can be considered constant along the dot thickness $L$, if this thickness in much smaller than the skin depth at the pumping frequency $\delta\left(\omega_{p}\right)$.
The power $P_{\text {rad }}$ radiated by the dot is proportional to the area of the dot base $A=$ $V / L$ and, in accordance with the Newton's law ${ }^{17}$, to the change of the dot temperature $\Delta T$ caused by the microwave heating:

$$
P_{\text {rad }}=\frac{\partial Q_{-}}{\partial t}=\beta \frac{V}{L} \Delta T
$$

where $\beta$ is the coefficient of heat exchange (heat transfer coefficient) between the dot and the substrate measured in $\mathrm{W} /\left(\mathrm{K}^{2} \mathrm{~m}^{2}\right)$. Solving equations $(2-4)$ the following expression is obtained for the temperature change of the magnetic dot:

$$
\Delta T=\frac{1}{2} \omega_{p} h_{p}^{2} L \frac{\chi^{\prime \prime}}{\beta}\left(1-e^{-\frac{t}{\tau_{T}}}\right),
$$

where the characteristic time $\tau_{T}$ of the dot heating is given by:

$$
\tau_{T}=\frac{c}{\beta} L
$$

It is clear from the solution of (5) and (6) that the $\Delta T$ induced by the microwave heating of the dot, and the characteristic time $\tau_{T}$ of this heating, are proportional to the dot thickness $L$. For the typical value of the specific heat of Permalloy $c \sim 410^{6}$ $\mathrm{J} /\left(\mathrm{K} \cdot \mathrm{m}^{3}\right)$ and the value of the heat exchange coefficient between the dot and the substrate of $\beta \sim 4 \cdot 10^{4} \mathrm{~W} /\left(\mathrm{K} \cdot \mathrm{m}^{2}\right)^{19}$ we get the characteristic time $\tau_{T} \sim 10 \mu$ s for the dot of the thickness $L=100 \mathrm{~nm}$, which agrees reasonably well with the characteristic time of power decrease in the dots of this thickness shown in Fig. 4. Since the change of the dot temperature (5) due to the microwave heating is proportional to the dot thickness $L$, the heating-related decrease of the power of sub-harmonic radiation is much less pronounced for the dots of smaller $(L=12 \mathrm{~nm})$ thickness (see upper curve in Fig. 4).

1. "Mesoscopic physics", McGraw-Hill Encyclopedia of Science and Technology, $10^{\text {th }}$ edition (McGraw-Hill Professional, 2007)

2. Nanostructured Materials: Processing, Properties and Applications, $2^{\text {nd }}$ edition Edited by C.C. Koch (William Andrew Publishing, New York, 2007).

3. Guslienko, K. Y. Magnetic Vortex State Stability, Reversal and Dynamics in Restricted Geometries. J. Nanosci. Nanotechn. 8, 2745-2760 (2008).

4. Demokritov, S. O. (Ed.) Spin wave confinement (Pan Stanford Publishing, Singapore, 2009)

5. Zivieri, R. \& Stamps, R. L. Theory of spin wave modes in tangentially magnetized thin cylindrical dots: a variational approach. Phys. Rev. B 73, 144422 (2006).

6. Gurevich, A. G. \& Melkov, G. A. Magnetization Oscillations and Waves (CRC Press, New York, 1996).

7. Morgenthaler, F. R. On the possibility of obtaining large amplitude resonance in very thin ferrimagnetic disks. J. Appl. Phys. 33, 1297-1299 (1962).

8. L'vov, V. S. Wave Turbulence under Parametric Excitation (Springer, Berlin, 1994).

9. Guslienko, K. Y. \& Slavin, A. N. Spin-waves in cylindrical magnetic dot arrays with in-plane magnetization. J. Appl. Phys. 87, 6337-6339 (2000).

10. Joseph, R. I. Ballistic Demagnetizing Factor in Uniformly Magnetized Cylinders. J. Appl. Phys. 37, 4639-4643 (1966).

11. Ulrichs, H., Demidov, V. E., Demokritov, S. O. \& Urazhdin, S. Parametric excitation of eigenmodes in microscopic magnetic dots. Phys. Rev. B 84, 094401 (2011).

12. Jorzick, J. et al. Spin wave wells in non-ellipsoidal micrometer size magnetic elements, Phys. Rev. Lett. 88, 047204 (2002).

13. Louisell, W. H. Coupled Mode and Parametric Electronics (John Wiley \& Sons. Inc., New York, 1960).

14. Chumak, A. V. et al. Parametrically stimulated recovery of a microwave signal using standing spin-wave modes of a magnetic film. Phys. Rev. B 79, 014405 (2009).

15. Melkov, G. A. et al. Nonlinear interactions of spin waves with parametric pumping in permalloy metal films, Phys. Rev. B 79, 134411 (2009).

16. Tiberkevich, V.S. \& Slavin, A. N. Nonlinear phenomenological model of magnetic dissipation for large precession angles: generalization of the Gilbert model. Phys. Rev. B 75, 014440 (2007).

17. Carslaw, H. S. \& Jaeger, J. C. Conduction of Heat in Solids, Second edition (Oxford University Press, Oxford, 1959).

18. Mangiaracina, R. S. \& Stern E. Ferrite high power effects in waveguides. IRE Trans. on Microwave Theory and Techniques 7, 11-15 (1959).

19. Guenin, Bruce. Transient Modeling of a High-Power IC Package, Part \#1. Electronics Cooling 17, 12 (December 2011).

\section{Acknowledgements}

Y.K., G.M. and A.S. acknowledge support from the U.S. National Science Foundation, DARPA, and the U.S. Army TARDEC, RDECOM, the State Fund for Fundamental Researches of Ukraine (Project \# UU34/008) and the Australian Research Council, K.G. acknowledges support by IKERBASQUE (the Basque Foundation for Science) and by MEC Grants PIB2010US-00153 and FIS2010-20979-C02-01. Work at Argonne was supported by the U.S.DOE Office of Science under Contract No.DE-AC02-06CH11357. M.K. acknowledges support by the Australian Research Council. 


\section{Author contributions}

V.N. and M.K. fabricated samples. Y.K. and G.M. performed measurements and data analysis. G.M., A.S. and K.G. developed the theoretical interpretation of the results. G.M., A.S., K.G. and S.D.B. co-wrote the text of the manuscript. All the authors discussed the obtained results and commented on the manuscript.

\section{Additional information}

Competing financial interests: The authors declare no competing financial interests. License: This work is licensed under a Creative Commons

Attribution-NonCommercial-ShareAlike 3.0 Unported License. To view a copy of this license, visit http://creativecommons.org/licenses/by-nc-sa/3.0/

How to cite this article: Kobljanskyj, Y. et al. Nano-structured magnetic metamaterial with enhanced nonlinear properties. Sci. Rep. 2, 478; DOI:10.1038/srep00478 (2012). 